D O C U M E N T O

\title{
La Cartagenidad: Un Sentimiento Colectivo de Ciudad, una Propuesta para el Fortalecimiento de lo Público Y LA RECONSTRUCCIÓN SOCIAL DE Cartagena de Indias*
}

\author{
Por: Fredi Eduardo Goyeneche González
}

\begin{abstract}
Cuando fundamos nuestros corazones con el alma del pueblo, seguramente perderemos el nombre, pero habremos ganado la eternidad. Antonio Machado
\end{abstract}

\section{RESUMEN}

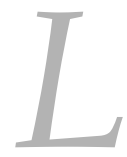

a idea de desarrollar propuestas con origen en ejercicios de participación libre de ciudadanos frente al concepto de lo público, condujo al redactor de este documento a orientar una tarea de conjugar y concretar iniciativas razonadas y argumentadas desde las perspectivas de actores de diversos intereses o creencias sociales, económicos, religiosos, políticas, culturales o tan solo en su condiciones de ciudadanos anónimos utilizando como metodología dos mecanismos: uno, el encuentro de estos actores en conversatorios alrededor de tres elementos: la percepción cotidiana, los planes de desarrollo desde la institucionalización de la elección popular de los alcaldes y las estadísticas básicas de Cartagena en 1999. El otro, escuchar las reflexiones de algunos actores de los mismos sectores pero escogidos de forma totalmente al azar y dependiendo de su disposición. Lo que se presenta a continuación es el resultado de ese ejercicio.

\section{Presentación}

El inicio del nuevo milenio sorprende a la ciudad en condiciones de profunda

* Este documento es resultado de reflexiones colectivas adelantadas por académicos interesados en problemáticas de la ciudad de Cartagena de Indias. Para su publicación se hicieron algunos recortes editoriales del documento original. Cartagena de Indias, Julio 11 de 2000. fragmentación social, con niveles de desempleo sin precedentes en la historia reciente, con gran decrecimiento de la actividad económica local, con altísimos niveles de contaminación y vulnerabilidad de los diversos ecosistemas que ponen en peligro su sostenibilidad ambiental, con un sistema administrativo de lo público evidentemente ineficiente, ineficaz, con poca transparencia y reiterativa crisis financiera y fiscal. Los indicadores que señalan el comportamiento de las infracciones a la ley y a la integridad del ciudadano y sus propiedades, muestran 
el deterioro creciente de la seguridad ciudadana. Las responsabilidades de la Administración Pública de la ciudad no dan cuenta de la salud y la educación pública, profundizando de esa manera la desigualdad y la inequidad social. Las constantes movilizaciones populares, cívicas y gremiales, que acuden a las vías de hecho y la inoperancia de las instancias legales de control, ilustran sobre la pérdida de credibilidad de las instituciones en el colectivo ciudadano.

A pesar de ser Cartagena pionera en la implementación de instancias desconcentradoras de la administración publica, el avance y las materializaciones efectivas en cabeza de sus Juntas Administradoras Locales no han sido significativas en el orden presupuestal o en la formulación de sus Planes de Desarrollo. Precisamente la formulación durante los últimos dos años del Plan de Ordenamiento Territorial ha desnudado toda la debilidad de su capital social. Muestra la ciudad, sin embargo, una ebullición de la participación espontánea y desordenada que sugiere intenciones de cambio que no encuentran orientación convergente. La ciudad tiene mil formas de reconocerse pero todas inconexas entre sí.

La importante y significativa penetración y adaptación a la vida local de inmigrantes, antes extranjeros y ahora nacionales, con sus aportaciones culturales y sus racionalidades ancestrales, muestran un sentimiento receptivo, hospitalario y generoso del nativo que se renueva en su ciudadanía cartagenera. El creciente desplazamiento de personas víctimas de la violencia y la recesión económica hacia Cartagena, de manera tan desordenada y espontánea que hace imposible su adaptación e integración al medio, es desde ya una de las más preocupantes realidades para la estabilidad social de la ciudad.

El surgimiento de organizaciones sociales, cívicas, populares, gremiales y de redes de veedurías ciudadanas, entre otras organizaciones, muestra el deseo de los ciudadanos de enfrentar la problemática común. Sin embargo estas soluciones están insufladas por el particularismo y la especificidad de intereses de los proponentes, como se infiere de las propuestas escuchadas y leídas en los diferentes foros que son cada vez más constantes y sobre diferentes tópicos. En los desencuentros entre estos actores, que se identifican y se reconocen como militantes contra un estado de cosas que los afecta a todos, aflora el sentimiento perverso que potencia la incapacidad para enfrentar solidariamente la problemática de la ciudad: la fragmentación social afecta poderosamente el sentido de la confianza pública. 


\section{Sentido y Orientación de la Propuesta}

El desconocimiento o no reconocimiento del ejercicio de la política como el ejercicio de lo público que nos involucra a todos y la pérdida del valor de lo público como espacio de encuentro a las aspiraciones colectivas que se concertan en la definición del bien común y el interés público, están en el núcleo de la reflexión y el debate por la construcción prospectiva de la ciudad.

Estamos ante la necesidad de redefinir los términos fundamentales para una sociedad cartagenera socialmente organizada en términos de equidad y de justicia, de economía productiva y de sostenibilidad ambiental. Frente a ello queda entonces como tarea fundamental una convocatoria de naturaleza ciudadana y democrática, que invocando los valores superiores de la cartagenidad -cultura, tradición, diversidad étnica-, la individualidad solidaria, la hospitalidad, la libre creatividad, y hasta la sabrosura y la bacanería desarrolle mancomunadamente el compromiso de rescatar a la ciudad de la grave y persistente situación de crisis que la mantiene estacionada en un marco de incertidumbre y de desconfianzas que no permiten la sinergia ciudadana y que no apalanca en la interacción social de sus

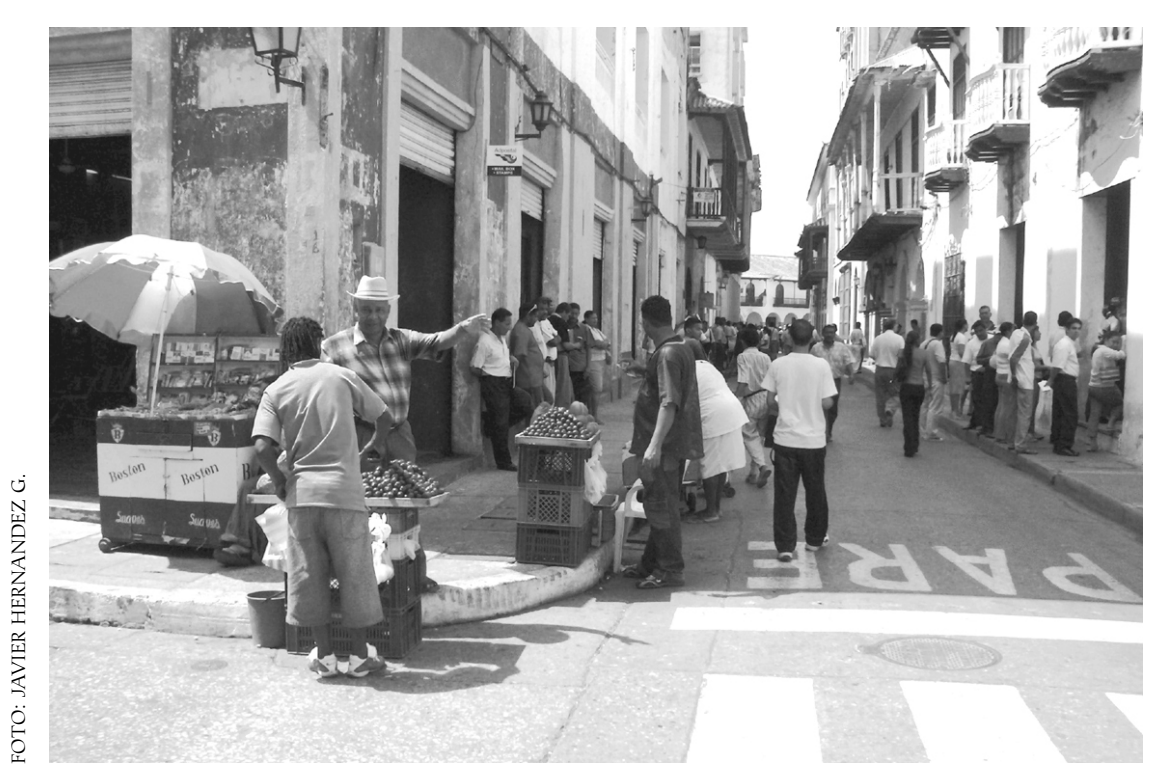

habitantes el incremento en la mejoría de las condiciones y la calidad de vida colectiva.

La propuesta de cartagenidad tiene dos sentidos. Uno, la definición de los criterios de democracia participativa, que debe seguir nuestra sociedad local en la intención de reconocerse como tal. Otro, la definición de unas líneas de gestión prioritaria que involucren los escenarios habituales del Distrito de Cartagena de Indias y sobre las cuales deben desplegarse todas las potencialidades de la gestión de gobierno.

En el primer sentido nuestra intención es fortalecer lo público. El concepto de lo público es etéreo y gaseoso en nuestra sociedad local. Los casi doscientos años de vida republicana no han sido, sin embargo, fortalecedores del ejercicio de la ciudadanía de una forma efectiva de manera que hubiese generado una fuerte y 
amplia gama de escenarios participativos de los ciudadanos que, por esta misma razón, encontraran familiares los escenarios de la política.

En el segundo sentido la orientación es de reconstruir socialmente a Cartagena. Ello supone no solo una lucha frontal y definitiva contra las desigualdades de todo tipo que generan las condiciones de conflictividad social. Ello debe involucrar el rescate de la confianza local no solo en sus gobernantes sino también en su propia auto estima de ciudadano. La construcción de lo público y de lo social debe considerar las propias potencialidades de la localidad y despertar desde allí la identidad sobre lo que es común.

La propuesta se perfila como un proceso continuo e inacabado de esfuerzos colectivos de los habitantes de la ciudad, con una visión social y prospectiva, tendiente a construir una sociedad cartagenera socialmente ordenada, justa, equitativa y organizada; con un ordenamiento territorial adecuado a sus potencialidades económicas, ambientales y culturales, y con la prioritaria necesidad de definir un sistema de administración y de finanzas públicas eficientes, eficaces y retributivas, todo ello teniendo como razón fundamental al habitante de la ciudad, el desarrollo de una ciudadanía definida como tal por el compromiso ético de la participación y el respeto colectivo por lo público.

Este objetivo nos lleva a la reflexión sobre la necesaria articulación del Fortalecimiento de lo Público y la Reconstrucción Social de Cartagena. Es preciso entonces, definir unas líneas básicas de gestión que permitan operacionalizar la propuesta en diferentes momentos y escenarios y que la hagan viable en el mediano y largo plazo.

\section{Fundamentos de la Gestión Programática}

Gobernar es fundamentalmente una función de interacción social en la cual las intenciones y propósitos de gobierno deben ser la interpretación más ética de la voluntad general, de tal forma que los gobernados sientan representadas sus demandas en los objetivos del gobernante. Atendiendo lo anterior proponemos como fundamentos de la gestión programática los siguientes:

Legitimidad: La legitimidad está relacionada con la conservación y fortalecimiento de la confianza de los ciudadanos en el gobierno que elige. Para que una Administración Pública concite el apoyo y pueda liderar las energías de los ciudadanos colectivamente en el logro de objetivos de gobierno debe adecuar su práctica política a los fines propuestos en su programa. 
Confianza Pública: No podrá ciertamente un conglomerado humano organizarse socialmente en términos de armonía social, libertades e igualdades si los sentimientos de dudas, incertidumbres y desconfianzas ocupan mucho espacio de sus cotidianidades. Es esta la mayor debilidad de la sociedad cartagenera y la que define la desafección ciudadana por lo público. La confianza pública es un fundamento absolutamente definitivo para garantizar el crecimiento, el desarrollo y la equidad de la riqueza social de Cartagena y sus habitantes.

Eficiencia: Un fundamento de una gestión signada por el buen gobierno debe considerar como norma de acción el cálculo de insumos en relación con el logro esperado. La eficiencia tiene que constituirse en inherente a la gestión, debe acompasar los proyectos con las acciones que estos requieran en término de productividad derivada de la relación costo-beneficio social

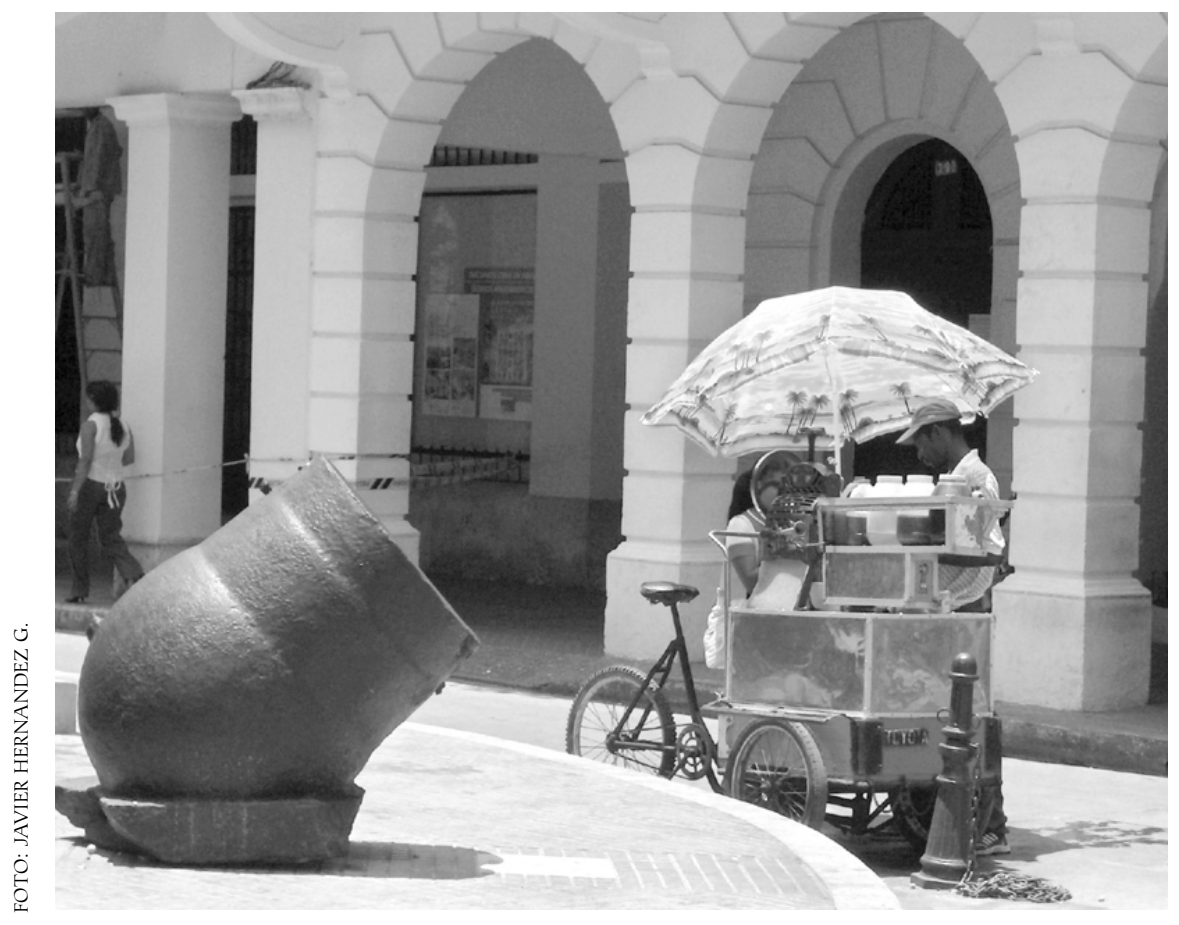

Efectividad: La administración debe conseguir lo que se propone. La acción de gobierno debe obtener los efectos y las metas con la menor desviación del curso de la acción y de los insumos requeridos. La efectividad en la concreción de los presupuestos programáticos en su especificidad de proyectos, aumenta la seguridad y la seriedad de la Administración para responder por las expectativas que generaron su elección pública.

\section{Líneas Básicas para los Frentes de Gestión de la Propuesta}

Estas líneas básicas son el señalamiento de áreas estratégicas de la vida social local, sobre las cuales tenemos que trabajar conjuntamente para que la voluntad general moldee la forma de su propia sociedad. Las líneas básicas tienen permanencia en cuanto muestran un escenario amplio de gestión que debe ser trabajado con especificidad en proyectos que tendrán términos y seguimiento en el tiempo. 


\section{- Construcción Institucionalizada y Colectiva de lo Público}

Si alguna razón pudiera explicar eficientemente el estado de desorden y desorganización social de Cartagena esta debe ser la de la ausencia de un sentido colectivo de lo público. Pero aun más grave, deducido por el comportamiento de los cartageneros en los últimos tiempos, es que también desde lo privado se desconoce su relación y limites con lo público, esto aparece claro en las siguientes situaciones que vive la ciudad.

¿Que significado tiene la invasión de calles y parques, de andenes, de plazas y en general de cualquier espacio público sino precisamente el no reconocimiento de éste? ¿No son acaso los conocidos acuerdos que aumentaban índices de construcción en beneficio particular de algunos constructores, y en perjuicio de toda la ciudad por las limitaciones de servicios públicos, una expresión que ilustra sobre el uso de lo público, el poder político, para beneficios privados?, ¿Cómo se explican, si no es bajo el desconocimiento de lo que es público, los escándalos por los manejos de una institución pública como lo es la Contraloría Distrital durante 1998 y 1999 ?

Cartagena vive una etapa de su vida ciudadana de mayor acentuación de la incertidumbre entre los límites de lo que es público y por lo tanto de todos, y lo que es privado y por lo tanto dependiente tan solo de la decisión autónoma del individuo. Rescatar y afianzar el sentido interiorizado en el ciudadano y en la conciencia colectiva de la sociedad cartagenera, del sentido de lo público debe fortalecer el capital social producto igualmente del crecimiento de la confianza del ciudadano en sus instituciones.

\section{Proyectos Tipo de esta Línea}

$\checkmark$ Proyectos que conduzcan al rescate de tradiciones y de la cultura local, de la cultura tradicional barrial, que permitan la pluralidad y la incorporación de culturas de otras regiones con el reconocimiento de los valores culturales propios. Para ello el Instituto de Cultura de Cartagena priorizará las actividades locales que tengan que ver con la cultura de la cartagenidad.

$\checkmark$ Proyectos que fortalezcan la participación de la sociedad civil en la elección de cargos de control. Por ejemplo garantizar audiencias públicas con participación de organizaciones sociales para el escogimiento del Contralor y del Personero Distrital, institucionalizar las redes de veeduría cívica. 
$\checkmark$ Proyectos que vigoricen la descentralización y desconcentración de la Administración Distrital considerando las Juntas Administradoras Locales y las organizaciones sociales. Que involucren la creación de instancias de participación en la planeación del Distrito tales como: Consejos Locales de Planeación, Escuelas Locales de Gobierno (ambas instancias por comunas ó zonas, a titulo de ejemplo).

$\checkmark$ Proyectos que potencien y efectivicen la participación de la sociedad civil en la formulación del Presupuesto Distrital. Que faciliten el sistema de rendición de cuentas ante la sociedad civil.

$\checkmark$ Proyectos que ordenen y reglamenten el uso y disfrute de los espacios públicos.

\section{- Viabilidad y Autosostenibilidad Financiera del Distrito}

Cartagena ha venido soportando desde hace por lo menos diez años, una recurrente situación de déficits presupuestales, fiscales y de tesorería. El crecimiento desbordado de su capacidad de crédito y el servicio de esta deuda, acompañado con una estructura de gastos que no guarda relación simétrica con la generación de ingresos se encuentran en el núcleo de esta problemática.

Enfrentar esta grave situación significa un replantamiento fiscal severo en el corto y mediano plazo que permita su autosostenibilidad financiera a largo plazo. Para ello es necesario determinar las prioridades de inversión y de sostenimiento de la carga administrativa, adecuando ambos a la importancia que tendrá esta gestión en el saneamiento financiero.

El Distrito, como ente territorial y como persona jurídica, auscultará las posibilidades financieras y económicas que puedan derivarse de desarrollos productivos empresariales en los cuales las fórmulas de la economía mixta puedan retribuir la utilización de las potencialidades de nuestra territorialidad Cartagena.

\section{Proyectos Tipo de esta Línea}

$\checkmark$ El Presupuesto Distrital será efectivamente un instrumento racionalizado de planeación por excelencia. No habrán presupuesto desbalanceados o equilibrados artificialmente. El equilibrio presupuestal será dogma en su formulación.

$\checkmark$ Defensa de los activos distritales y su valoración con intenciones no de privatización, sino como recurso productivo del Distrito. 
$\checkmark$ Proyecto que redefina la estructura tributaria ajustándola a las condiciones de la economía local y que elimine las exenciones tributarias.

$\checkmark$ Proyecto que defina unas mas justas relaciones financieras y de beneficio para la ciudad del contrato de concesión con Aguas de Barcelona.

$\checkmark$ Proyecto que defina los términos de distribución de participación en las concesiones desprendidas de la privatización de los puertos (ley $1^{\mathrm{a}}$ de 1991).

$\checkmark$ Proyecto que, en cumplimiento de normas constitucionales y en particular de la ley 388 de 1997, permita legalizar los baldíos como fuente de ingresos fiscales para el Distrito.

$\checkmark$ Proyecto que permita una más adecuada y justa liquidación del impuesto predial, ajustada a la situación económica local, pero que efectivamente derive en una fuente eficiente de recursos para el Distrito, sin poner en peligro la estabilidad de la propiedad de los inmuebles.

$\checkmark$ Proyecto que permita controlar la evasión fiscal a través de medidas combinadas de cohersión, estimulo y facilidades mas no las amnistías en ninguna forma.

$\checkmark$ Proyecto tendiente a simplificar los tramites de cobro y pago de las obligaciones fiscales, sistematizando y conectando sistemas de evaluación y control de información con entidades como la Cámara de Comercio y la Dian.

$\checkmark$ Proyectos tendientes a facilitar la inversión en proyectos tales como la construcción de la Planta de Olefinas, para lo cual se harán gestiones ante el Gobierno Central, que propicien las condiciones para este tipo de inversión del capital privado.

\section{- Sostenibilidad Ambiental del Distrito}

Cartagena es tal vez una de las ciudades más frágiles desde el punto de vista de su sostenibilidad ambiental. Su condición de ciudad industrial, portuaria y turística ha contribuido paradójicamente a una depredación mayor de su entorno natural por un crecimiento desplanificado de su oferta y demanda de servicios en derivadas de sus vocaciones mencionadas.

\section{Proyectos Tipo de esta Línea}

$\checkmark$ Proyectos de la naturaleza del Plan Maestro de Acueducto y 
Alcantarillado serian revisados y ajustados, si fuera necesario, obedeciendo criterios técnicos, financieros, la opinión de usuarios y de la ciudadanía en general.

$\checkmark$ Fortalecer las gestiones oficiales y locales tendientes a continuar y finalizar el saneamiento de los cuerpos de agua internas, si es posible considerando nuevas formas de financiamiento, incluida la concesión privada

$\checkmark$ Proyectos como los del emisario submarino de aguas servidas, serán rigurosamente sometidos a todas las discusiones posibles con organizaciones de ciudadanos vecinos al posible punto de ubicación del emisario, organismos especializados, asociaciones del sector turístico y económico y en general con todos los actores sociales que tengan a bien participar y aportar en esta gran decisión colectiva.

$\checkmark$ Proyectos tendientes a fortalecer la conciencia ambiental en la población cartagenera que incluyan la formación desde la escuela, la creación de Cabildos Verdes, el estimulo a la creación de organizaciones ambientalistas que cumplan funciones cívicas en sus localidades.

$\checkmark$ Proyecto tendiente a crear el Sistema Ambiental de Cartagena, el cual articularía toda la administración en sus diferentes instancias coordinados por que cumpliría una función a su vez de enlace con Cardique como autoridad ambiental de la región.

$\checkmark$ Proyectos y gestión que hagan posible que los departamentos de origen de los desplazados por la violencia o desastres naturales, desplacen igualmente al Distrito los recursos de la nación que reciben para el sostenimiento de esta población.

$\checkmark$ Proyectos que con fuerte participación de las comunidades beneficiadas, avance en la solución de reubicaciones de viviendas en zonas de alto riesgo o en zonas inadecuadas para la vivienda.

\section{- Estructura Administrativa Formalizada y Adecuada para una Gestión Eficiente, Eficaz, Participativa y Transparente del Distrito}

Cartagena debe tener una estructura administrativa adecuada a las necesidades de la gestión de gobierno con criterios de desconcentración, descentralización, eficiencia, eficacia, participativa y transparencia. La legitimación misma del gobierno local depende en mucho de una efectiva presencia de este en todo el territorio del Distrito.

El control ciudadano es mucho más efectivo cuando la Administración transita 
cada día lo más cercana posible a la vida de las comunidades locales, materializada en sus actos y en sus funcionarios, sobre todo si son estos como seria lo ideal, habitantes de la propia zona. Esto significaría una fuente de relegitimación constante de la Administración y una razón suficientemente estimuladora de virtudes ciudadanas como la confianza pública

\section{Proyectos Tipo de esta Línea}

$\checkmark$ Proyectos tendientes a diseñar una estructura administrativa técnica adecuada a las necesidades y demandas de administración y gobierno del Distrito, considerando básicamente la descentralización y los gobiernos locales como los ejes rectores de esta nueva estructura. Esta estructura tendrá desde la Administración Central, funciones aglutinantes por afinidades en los frentes de gestión en gobierno, finanzas y economía pública, social, infraestructura y competitividad. En lo local, la estructura se debe expresar en términos de Secretarias Locales de Desarrollo que hagan prácticas y efectivas las determinaciones de acción en cada frente de la Administración Central.

$\checkmark$ Proyecto tendiente a crear institucionalmente la Escuela de Gobierno de Cartagena de Indias, abierta a la investigación y profundización en el conocimiento de temas de la Administración Pública.

$\checkmark$ Proyectos tendientes a establecer de manera permanente la capacitación en gobierno y administración pública en las Comunas de la ciudad con la participación de las organizaciones cívicas y sociales de cada localidad.

\section{- Planeación Integral del Desarrollo y el Ordenamiento del Territorio del Distrito}

Esta línea marca el punto de partida para el logro del desarrollo del sentimiento de cartagenidad, que debe ser el pegamento social de nuestra sociedad local. Aquí se condensa la integralidad pero se estimula la diversidad creativa.

La planeación del desarrollo económico, social y del ordenamiento territorial del Distrito hay que convertirlo en el escenario natural y perenne del fortalecimiento de lo público y el insumo definitorio de su reconstrucción social. De allí que la propuesta de esta línea de gestión apunte a construir instancias de encuentro para el debate, el disenso y el consenso como mecanismos validadores y legitimantes de los acuerdos planificadores. 


\section{Proyectos Tipo de esta Línea}

$\checkmark$ Proyectos tendientes a crear un sistema de planeación económica, social y territorial de la ciudad, que inicie y se retroalimente desde lo local, con la participación de los actores relevantes de la vida de las localidades, y demás actores sociales y económicos.

$\checkmark$ Proyectos tendientes a crear espacios institucionalizados para el desarrollo de pensamientos y saberes en cuanto a la administración y planeación de la ciudad tales como Escuelas de Gobierno, Centros de Pensamiento, Consejos Locales de Planificación, Audiencias Públicas, Cabildos Abiertos. La intención es desarrollar toda una cadena ciudadana que se preocupe y ocupe por estas actividades, como parte de sus responsabilidades sociales.

$\checkmark$ Proyectos tendientes a condensar en un solo aparato administrativo las actividades financieras y fiscales del Distrito con las de planificación del desarrollo económico, social y territorial del mismo.

$\checkmark$ Proyectos tendientes a institucionalizar los mecanismos de rendición de cuentas de la Administración de forma periódica, institucionalizando igualmente el voto de censura por parte del Concejo Distrital y de las comunidades organizadas.

\section{- Generación de Condiciones Estimulantes para el Espíritu Empresarial y la Economía Competitiva}

Cartagena no ha aprovechado la apertura propuesta por el actual modelo económico. Las políticas públicas de las últimas administraciones distritales no han adelantado la gestión en este sentido. Estas políticas no han avanzado en la creación de condiciones estimulantes para la ubicación o reubicación empresarial en el Distrito de los inversionistas, tales como, las comunicaciones, la infraestructura de servicios y vial, la seguridad, la estabilidad en las normas que institucionalizan la actividad inversionista y sus responsabilidades sociales y fiscales, entre las más importantes.

Cartagena debe adecuar la calidad de su recurso humano a las demandas cambiantes de las nuevas oportunidades. En un ambiente de alta competencia económica, la más importante de todas las estrategias debe estar dirigida a desarrollar potencialidades, creatividades e innovaciones en la oferta de la mano de obra cartagenera. 


\section{Proyectos Tipo de esta Línea}

$\checkmark$ Proyectos tendientes a reorganizar el sistema educativo local centrando el control de la administración de este servicio en un tipo de organización tripartita: padres de familias, docentes y educandos, que velen por la calidad académica, el control de matriculas y la contratación.

$\checkmark$ Proyectos que involucren a través de convenios u otros mecanismos, a la Universidad de Cartagena como consultora, asesora y ejecutora de estudios, obras civiles y en general todos los campos del saber y de la técnica.

$\checkmark$ Proyectos tendientes a revisar el estado actual de nuestra estructura de servicios de salud pública, incluyendo las recientemente constituidas Empresas Sociales del Estado, con el fin de establecer demandas por servicios y factibilidad de las futuras condiciones de sostenibilidad financiera de estas ESE.

$\checkmark$ Proyectos tendientes a redefinir los usos de la bahía, correspondiendo estos a la interrelación entre los cuerpos de agua y su entorno, las características físicas del territorio y el uso racional de este desde lo económico, social, cultural y ambiental.

$\checkmark$ Proyectos tendientes a la reubicación de los muelles de carga y astilleros de la bahía interna facilitando administrativamente su localización en la bahía externa, que será definida como de uso portuario e industrial. Para la bahía interna debe quedar definido su uso como histórico - monumental, turístico, marina y de deportes náuticos.

$\checkmark$ Proyectos tendientes a articular una red vial en correspondencia a la vocación industrial, portuaria y turística de Cartagena. Esta red de vías contempla los diferentes medios que puedan articuladamente producir la mejor utilización y manejo del tiempo desde el punto de vista de la productividad y la humanización de los procesos productivos.

$\checkmark$ Proyectos tendientes a formular un sistema de transporte público terrestre masivo que permita armonizar impactos mínimos desde lo ambiental al aumento de la productividad y la humanización del servicio.

$\checkmark$ Proyectos orientados a lograr articular nuestro sistema de caños y lagunas al sistema vial intraurbano, organizando el servicio para ser ejecutado por sistema de concesión a inversionistas privados.

$\checkmark$ Proyectos tendientes a la incorporación de Cartagena a un sistema 
ferroviario que articule nuestra ciudad al corredor caribe y al resto de la república aumentando la productividad y disminuyendo costos para nuestra oferta productiva

$\checkmark$ Proyecto tendiente a organizar empresarialmente un sistema de mercados de productos básicos y de nuestra cultura gastronómica, con el fin de garantizarle mercados a los productos de nuestras zonas rurales.

$\checkmark$ Proyectos tendientes a organizar la actividad económica en el frente turístico, en la cual la función de la Administración será de carácter coordinador. La Administración buscara medios diversos para tales fines, incluidas las concesiones, privilegiando las organizaciones sociales de oferentes de servicios turísticos, tales como cooperativas y sindicatos.

\section{Comentarios Finales}

Hay que partir de la premisa que lograr entre los ciudadanos el sentido de lo público, es un proceso que debe tener como base una recomposición social de la ciudad con un sentido igualitario y equitativo por una parte, y de otra, traducirse igualmente en mejores condiciones objetivas para desarrollar el espíritu empresarial y la competitividad.

Esta propuesta debe concretarse en programas de gobierno que lo irán avanzando en el corto, mediano y largo plazo apoyados en un proceso de interacción social signado por la participación de la comunidad cartagenera. Lo que debemos es profundizar este debate con una nutrida participación de la sociedad civil en un escenario de disenso sincero, creativo y tolerante, que nos permita conjugarnos como una sociedad de diferencias armonizadas en un clima de respeto por la dignidad del otro, del diferente ó del que piensa razonablemente diferente a la mayoría pero pensando con sentimiento de cartagenidad.

BIOGRAFÍA

FREDI GOYENECHE GONZALEZ

Economista (Universidad de Cartagena), Docente Universidad de Cartagena. Especialista en Planeación del Desarrollo Rural y Urbano (Universidad de Cartagena), Gestión Pública (ESAP- UNICARTAGENA) y Candidato a Maestría Estudios Políticos y Económicos (Universidad del Norte). 